\title{
Acute Adult Supraglottitis: An Impending Threat to Patency of Airway and Life
}

\author{
Nissar Shaikh ${ }^{1}$, Shoaib Nawaz ${ }^{1}$, Kiran Ahmad $^{1}$, Muna Al Maslamani ${ }^{2}$ \\ 1. Anesthesia, Hamad Medical Corporation, Doha, QAT 2. Infectious Diseases, Hamad Medical Corporation, Doha, QAT
}

Corresponding author: Shoaib Nawaz, shoaibnawaz89@hotmail.com

\begin{abstract}
Introduction

Acute adult supraglottitis (AAS) is one of the upper airway infections that can potentially cause upper airway obstruction and, if not treated promptly, can be life-threatening. The widespread use of vaccines against Hemophilus influenzae has decreased the incidence of epiglottitis in children, whereas the incidence of AAS is on the rise. We aim to highlight the presentation, diagnosis, and management in AAS with our study.
\end{abstract}

Patients and Methods

A retrospective analysis was performed on all patients admitted to a tertiary health care facility surgical intensive care unit (SICU) where AAS was identified and the demographic data, duration of symptoms, imaging studies, management, and complications were recorded. In these patients, the diagnosis of AAS was confirmed by nasopharyngeal endoscopy. Data was entered in the IBM Statistical Package for Social Sciences (SPSS), version 23 (IBM SPSS Statistics, Armonk, NY), and groups were compared using student t-test and chi-square test. $\mathrm{P}$ values of $\leqslant 0.05$ were considered statistically significant.

Results

A total of 118 patients were admitted to the SICU. The male: female ratio was 3.9: 1. Major risk factors were smoking and drinking cold liquids. The common presenting symptom was sore throat (89.8\%). The thumb sign was positive in $65 \%$ of the patients. Common bacteria were the Streptococcus species (11.9\%). Ceftriaxone was the most commonly prescribed inpatient antibiotic. All patients received steroids as adjuvant therapy. Adrenaline nebulization was used in $66 \%$ of the cases. Forty-six percent of patients required endotracheal intubation. In $10.2 \%$ of patients, intubation was not possible and in $12.7 \%$ of patients, a tracheostomy was done. Ludwig's angina was the most frequent complication. Patients presenting with dysphagia and fever had a significantly higher incidence of Ludwig's angina $(\mathrm{P} \leqslant 0.02$ and 0.005 , respectively). AAS patients complicating into Ludwig's angina (severe cellulitis of submandibular, submental, and sublingual spaces) had a significantly longer duration of symptoms, a higher incidence of streptococcal infection, airway interventions, and prolonged stay in an intensive care unit $(\mathrm{p} \leqslant 0.05)$.

Received 07/24/2020

Review began $07 / 27 / 2020$

Review ended 08/03/2020

Published 08/23/2020

(๑) Copyright 2020

Shaikh et al. This is an open access article distributed under the terms of the Creative Commons Attribution License CC-BY 4.0., which permits unrestricted use, distribution, and reproduction in any medium, provided the original author and source are credited.
Conclusion

Male gender, smoking, and drinking cold liquids were the risk factors associated with AAS, and thumb sign on lateral neck soft tissue X-ray was suggestive of it. AAS caused by Streptococcus species was a relatively serious condition, leading to complications like Ludwig's angina.

Categories: Emergency Medicine, Otolaryngology, Infectious Disease

Keywords: apnea, dysphagia, dysphonia, ludwig's angina, sore throat, streptococcal species, tracheostomy, adult acute supraglottitis (aas)

\section{Introduction}

Acute adult supraglottitis (AAS) used to be called epiglottitis in the past. Supraglottitis is the more appropriate terminology in adults in contrast to the epiglottitis in children where the infection commonly involves only epiglottis. AAS is not only infection and inflammation of epiglottis but also of the surrounding structures namely arytenoid, uvula, the false vocal cords, and laryngeal ventricles [1-2]. Acute adult supraglottitis (AAS) is a serious and potentially life-threatening disease causing airway obstruction which, if not diagnosed early and treated promptly, can lead to high morbidity and mortality. [1] In the past, bacterial epiglottitis was common in children and was caused by Haemophilus influenzae type b (Hib). By the strict implementation of nationalized vaccine programs against $\mathrm{H}$. influenzae in children, the rate of epiglottitis decreased in children from 99 to one case in 100,000. At the same time, the incidence of supraglottitis in the adult population increased from 0.88 to 3.1/100,000 patients [2]. An even higher incidence $(4.7 / 100,000)$ of AAS was reported in Finland [3]. The literature from this region about AAS is not available, and as per most 
international studies, the majority of the patients, including milder cases of supraglottitis, are managed in the intensive care setup.

\section{Materials And Methods}

After obtaining permission from the Institutional Medical Research Committee, Hamad Medical Corporation, Doha, all patients admitted to the surgical intensive care unit (SICU) of the only tertiary health care centre and university hospital during the period from January 2008 to December 2018 were identified from the register and included in the study. The demographic data, comorbidities, history of smoking, tobacco chewing, the recent history of ice-cold fluid intake (cold drinks/ice cream/etc.), duration of symptoms, initial signs and symptoms upon presentation, imaging studies, microbiology, antibiotic therapy, airway interventions and management, length of ICU (intensive care unit) stay, complications, and outcomes of all patients were recorded.

In all patients, the diagnosis of supraglottitis was confirmed by fiberoptic nasopharyngeal endoscopy (performed by the otolaryngology on-call specialist) which showed swelling, edema, inflammation, and infection of the epiglottis and surrounding structures. Exclusion criteria were all paediatric patients, adult patients who sustained trauma, patients who ingested corrosive liquids, and patients with radiotherapyinduced supraglottitis.

In our patient population, dysphagia was defined as difficulty in swallowing, dysphonia as a change in speech tone (muffled and/or hoarseness), sore throat as severe pain in the throat, stridor as noisy inspiration, and dyspnea as difficulty in breathing. Fever was defined as a temperature of more than $38.3^{\circ} \mathrm{C}$ (taken either from the oral cavity or forehead infrared scan) and leucocytosis when the white blood cell (WBC) count was more than $11 \times 10^{3}$ cells/ $\mu \mathrm{L}$. A throat swab was taken for microbiological evaluations. Others in microbiology included Escherichia coli (E. coli), anaerobes, Acinetobacter, and methicillin-resistant Staphylococcus aureus (MRSA).

Data were entered and analysed using the IBM Statistical Package for Social Sciences (SPSS), version 23 (IBM SPSS Statistics, Armonk, NY). Categorical variables were reported using numbers (n) and percentages (\%). Continuous variables were reported as mean \pm standard deviation (SD). The Kolmogorov-Smirnov test proved these variables to be normally distributed. Comparisons between groups were performed by using the chi-square test for categorical variables and the Student t-test for continuous variables. A two-tailed pvalue of $\leqslant 0.05$ was considered statistically significant.

\section{Results}

During the study period, a total of 118 patients were admitted to the SICU with acute adult supraglottitis (AAS). The majority of our patients were male (94/79.7\%) with a male: female ratio of 3.9: 1 (Table 1). The majority of comorbid conditions in our patients included diabetes mellitus in 35 (29.7\%) and hypertension in 34 (28.8\%) patients. Fifty-one patients (43.2\%) had a history of smoking and 49 patients (41.5\%) had a recent history of drinking ice-cold liquids, including drinks with added ice cubes and ice cream. The most common presentation of AAS was sore throat in 106 patients (89.8\%), followed by fever in 103 patients (87.3\%), dysphonia in 103 patients (87.3\%), stridor in 102 (86.4\%), dysphagia in 97 (82.2\%), and dyspnea in 69 patients (58.5\%). Leucocytosis was present in the majority of AAS patients (103 patients, 87.3\%) (Table 1). The thumb sign on lateral neck soft tissue $\mathrm{x}$-ray was positive in the majority of patients (77 patients, 65.2\%), although an x-ray was not done in 25 patients (21.2\%) (Table 2). Computerized tomography (CT) scan of the neck was performed in 53 patients (44.9\%) in which the diagnosis was not clear on presentation. The CT scan was done within 24 hours of presentation. A microbiological evaluation was not done in the majority of patients (87 patients, 73.1\%). Common microbial growth was streptococcal (14 patients, $11.9 \%$ ), followed by H. influenzae (five patients, $4.2 \%$ ), Klebsiella pneumoniae (K. pneumoniae) (five patients, $4.2 \%$ ), Candida albicans (three patients, $2.5 \%$ ), and others (four patients, 3.3\%). The commonly used antimicrobial agent was ceftriaxone, followed by piperacillin + tazobactam in 20 (16.9\%), amoxicillin + clavulanic acid in $12(10.2 \%)$, meropenem in six (5.1\%), azithromycin in five (4.2\%), and antifungals (anidulafungin) in three patients (2.5\%). Adrenaline nebulization was used in 78 patients $(66.1 \%)$. Steroids were used in all of our patients. Dexamethasone was the most commonly prescribed steroid that was used in 100 patients (84.7\%). Other steroids prescribed were hydrocortisone (16 patients, $13.6 \%$ ) and methylprednisolone (two patients, $1.7 \%)$. Sixty patients (50\%) did not require any airway interventions, 46 patients (39\%) required intubation using video laryngoscopy or fibrotic bronchoscopy (as they were difficult to intubate), and in 12 patients (10.2\%), intubation was not possible. Tracheostomy was required in 15 patients (12.7\%) (Table 2). There was no mortality in our case series. 


\section{Cureus}

\begin{tabular}{|c|c|c|c|}
\hline \multicolumn{2}{|l|}{ Variable } & Number & Percentage \\
\hline \multirow{2}{*}{ Gender } & Female & 24 & 20.3 \\
\hline & Male & 94 & 79.7 \\
\hline \multirow{2}{*}{ HTN (hypertension) } & Yes & 34 & 28.8 \\
\hline & No & 84 & 71.2 \\
\hline \multirow{2}{*}{ DM (diabetes mellitus) } & Yes & 35 & 29.7 \\
\hline & No & 83 & 70.3 \\
\hline \multirow{2}{*}{ Smoking } & Yes & 51 & 43.2 \\
\hline & No & 67 & 56.8 \\
\hline \multirow{2}{*}{ Cold drinks } & Yes & 49 & 41.5 \\
\hline & No & 69 & 58.5 \\
\hline \multirow{2}{*}{ Sore throat } & Yes & 106 & 89.8 \\
\hline & No & 12 & 10.2 \\
\hline \multirow{2}{*}{ Dysphonia } & Yes & 103 & 87.3 \\
\hline & No & 15 & 12.7 \\
\hline \multirow{2}{*}{ Fever } & Yes & 103 & 87.3 \\
\hline & No & 15 & 12.7 \\
\hline \multirow{2}{*}{ Leucocytosis } & Yes & 103 & 87.3 \\
\hline & No & 15 & 12.7 \\
\hline \multirow{2}{*}{ Stridor } & Yes & 102 & 86.4 \\
\hline & No & 16 & 13.6 \\
\hline \multirow{2}{*}{ Dysphagia } & Yes & 97 & 82.2 \\
\hline & No & 21 & 17.8 \\
\hline \multirow{2}{*}{ Dyspnea } & Yes & 69 & 58.5 \\
\hline & No & 49 & 41.5 \\
\hline
\end{tabular}

TABLE 1: Descriptive Data 


\section{Cureus}

\begin{tabular}{|c|c|c|c|}
\hline \multicolumn{2}{|l|}{ Variables } & Number & Percentage \\
\hline \multirow{3}{*}{ Thumb Sign } & Positive & 77 & 65.2 \\
\hline & Negative & 16 & 13.6 \\
\hline & X-ray not done & 25 & 21.2 \\
\hline \multirow{2}{*}{ Computed Tomography (CT) Neck } & Yes & 53 & 44.9 \\
\hline & No & 65 & 55.1 \\
\hline \multirow{6}{*}{ Microbiology } & Not done & 87 & 73.1 \\
\hline & Streptococcus species & 14 & 11.9 \\
\hline & H. influenzae & 5 & 4.2 \\
\hline & K. pneumoniae & 5 & 4.2 \\
\hline & Candida species & 3 & 2.5 \\
\hline & Others (E. coli, MRSA, Anaerobes, Acinetobacter) & 4 & 3.3 \\
\hline \multirow{6}{*}{ Antimicrobial Agents } & Ceftriaxone & 72 & 61 \\
\hline & Piperacillin + Tazobactam & 20 & 16.9 \\
\hline & Amoxicillin + Clavulanic Acid & 12 & 10.2 \\
\hline & Meropenem & 6 & 5.1 \\
\hline & Azithromycin & 5 & 4.2 \\
\hline & Antifungal & 3 & 2.5 \\
\hline \multirow{2}{*}{ Adrenaline Nebulization } & Yes & 78 & 66.1 \\
\hline & No & 40 & 33.9 \\
\hline \multirow{3}{*}{ Steroids } & Dexamethasone & 100 & 84.7 \\
\hline & Hydrocortisone & 16 & 13.6 \\
\hline & Methylprednisolone & 2 & 1.7 \\
\hline \multirow{3}{*}{ Endotracheal Intubation } & Not intubated & 60 & 50.8 \\
\hline & Not possible & 12 & 10.2 \\
\hline & Intubated & 46 & 49 \\
\hline \multirow{2}{*}{ Tracheostomy } & Yes & 15 & 12.7 \\
\hline & No & 103 & 87.3 \\
\hline
\end{tabular}

TABLE 2: Imaging Findings, Microbiology, and Treatment of Acute Adult Supraglottitis (AAS)

MRSA: Methicillin-resistant Staphylococcus aureus

Table 3 shows the patients' age, duration of symptoms, and complications. The mean age of the patients was 43.91 years. The mean duration of symptoms and length of ICU stay were 2.92 and 6.59 days, respectively. In 47 patients (38.8\%), there were no complications. The common complication in our AAS population was the development of multiple abscesses or Ludwig's angina (bilateral spread of infection to the submandibular space and the sublingual space forming abscesses). However, the possibility of an odontogenic infection leading to swelling of the glottic area could not be ruled out. Twenty patients (16.9\%) had obstruction to the upper airway, leading to apnea (Table 3). 


\section{Cureus}

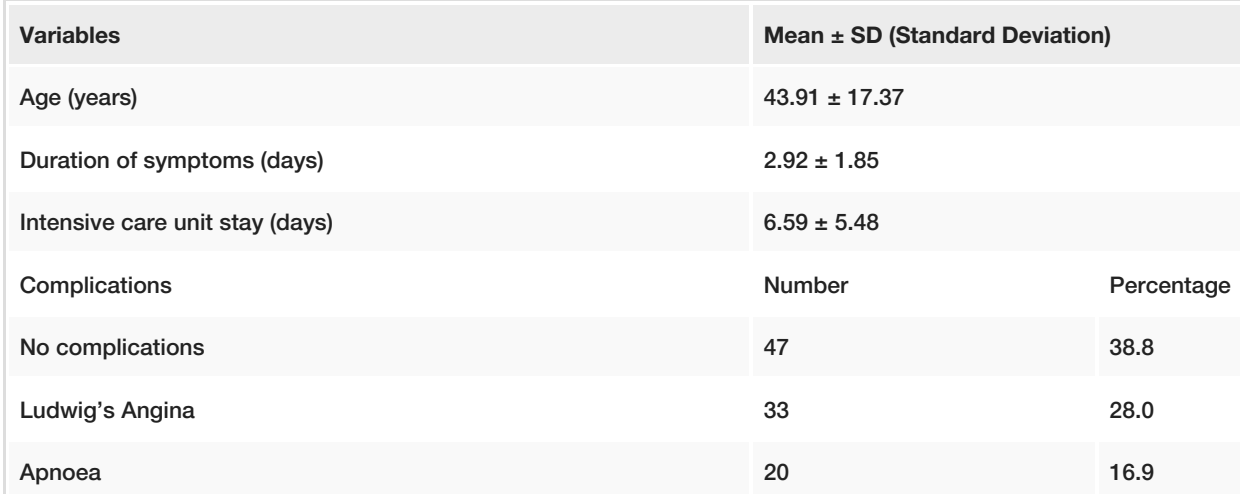

\section{TABLE 3: Patient Age, Durations, and Complications}

Table 4 shows variables associated with the complications of AAS leading to Ludwig's angina. The presence of fever and dyspnea are significantly associated with a prediction for Ludwig's angina $(\mathrm{p}=0.001$ and 0.005 , respectively). Patients with streptococcal infection had a significantly increased number of complications including Ludwig's angina $(p=0.001)$. AAS patients complicated by Ludwig's angina required a significant increase in airway interventions, both intubations and tracheostomies ( $\mathrm{p}=0.001$ and 0.006 , respectively).

Those patients had a longer duration of symptoms and a higher length of intensive care stay $(\mathrm{p}=0.001)$.

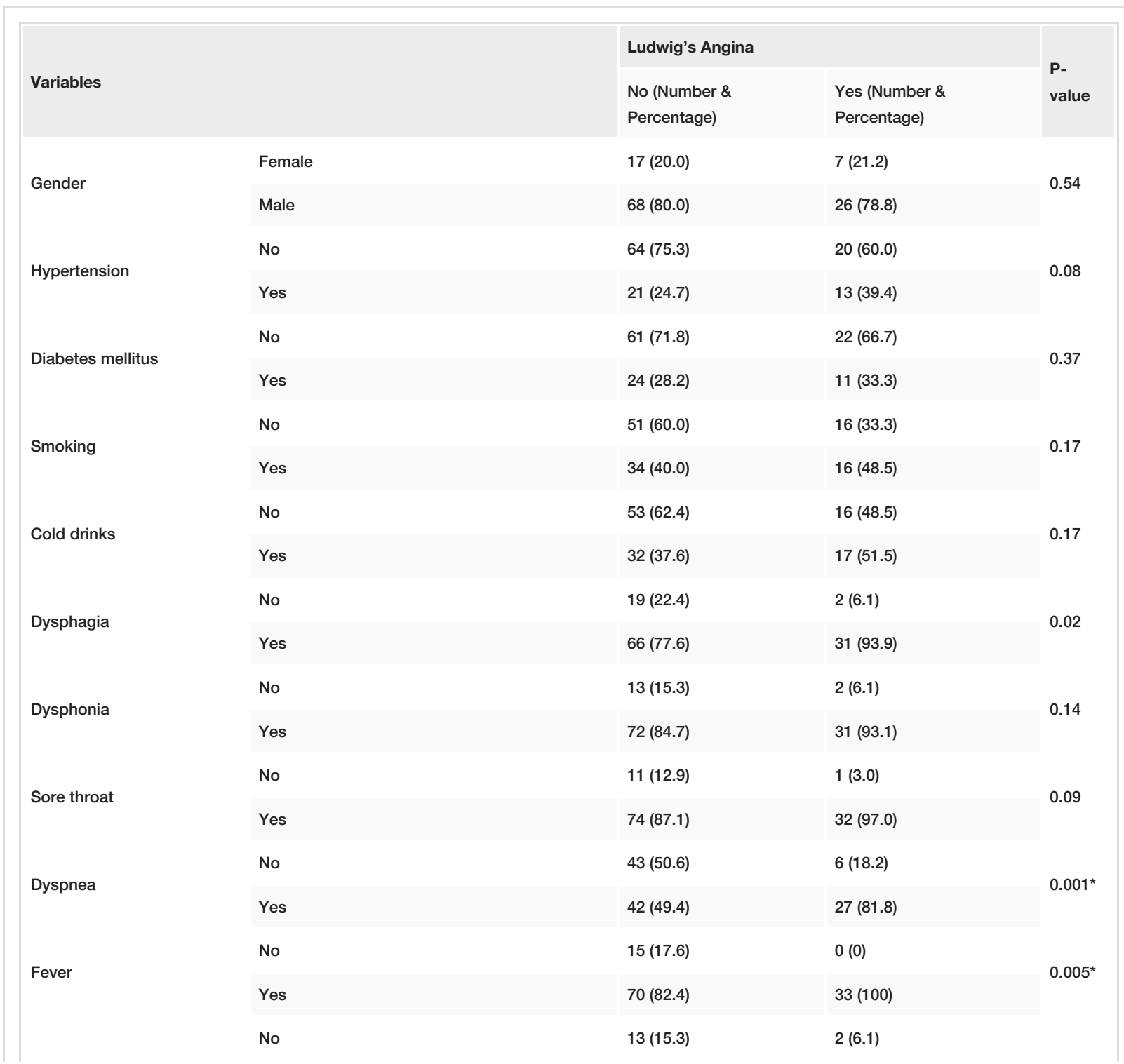




\section{Cureus}

\begin{tabular}{|c|c|c|c|c|}
\hline \multirow[t]{4}{*}{ Leucocytosis } & Yes & $72(84.7)$ & 31 (93.9) & \multirow[t]{2}{*}{0.14} \\
\hline & Not done & $74(87.1)$ & $13(39.4)$ & \\
\hline & Streptococcus & $3(3.5)$ & $11(33.3)$ & \multirow{5}{*}{$0.001^{*}$} \\
\hline & H. influenzae & $3(3.5)$ & $2(6.1)$ & \\
\hline \multirow[t]{4}{*}{ Bacteria (Culture) } & K. pneumoniae & $2(2.4)$ & $3(9.1)$ & \\
\hline & Fungus & $0(0)$ & $3(9.1)$ & \\
\hline & $\begin{array}{l}\text { Others (E. coli, anaerobes, MRSA, } \\
\text { Acinetobacter) }\end{array}$ & $3(9.1)$ & $2(6.1)$ & \\
\hline & Not Intubated & $59(69.4)$ & $1(1.7)$ & \multirow{3}{*}{0.001} \\
\hline \multirow[t]{2}{*}{ Intubation } & Not possible & $4(4.7)$ & $8(24.2)$ & \\
\hline & Intubated & $22(25.9)$ & $24(72.8)$ & \\
\hline \multirow{2}{*}{ Tracheostomy } & No & 79 (92.9) & $24(72.7)$ & \multirow{2}{*}{$0.006^{\star}$} \\
\hline & Yes & $6(7.1)$ & $9(27.3)$ & \\
\hline Variables & & Mean \pm SD & & $p$-value \\
\hline \multirow[b]{2}{*}{ Age (years) } & No & $43.59 \pm 18.43$ & & \multirow{2}{*}{0.13} \\
\hline & Yes & $44.73 \pm 14.52$ & & \\
\hline \multirow{2}{*}{$\begin{array}{l}\text { Duration of symptoms } \\
\text { (days) }\end{array}$} & No & $2.52 \pm 1.7$ & & \multirow{2}{*}{$0.001^{*}$} \\
\hline & Yes & $3.94 \pm 1.6$ & & \\
\hline \multirow{2}{*}{$\begin{array}{l}\text { Intensive care unit stay } \\
\text { (days) }\end{array}$} & No & $5.14 \pm 4.06$ & & \multirow{2}{*}{$0.001^{*}$} \\
\hline & Yes & $1033+682$ & & \\
\hline
\end{tabular}

\section{TABLE 4: Variables Associated With Ludwig's Angina}

\section{Discussion}

AAS is a potentially life-threatening infection due to the possibility of upper airway obstruction. Hence, these patients are preferably monitored in the intensive care environment [1-3]. AAS is a severe inflammation and infection of the epiglottis and surrounding structures. These structures have loose mucosa and a good blood supply; thus, the infection spreads quickly with a high risk of sudden fatal upper airway obstruction [3]. Ng et al. found that the AAS was more common in males with an M: F (male: female) ratio of 3: 1 [4]. Bizaki et al., however, reported a lower M: F ratio of 1.6: 1 in their review of 308 patients [3]. In our patient population, the M: F ratio was 3.9: 1 which may correspond to a generally higher male population in our country. There are various risk factors for the AAS mentioned in the literature, namely, diabetes mellitus, hypertension, obesity, smoking, alcohol abuse, pneumonia, and malignancies. The majority of AAS patients do not have any comorbidities [5-6]. In our patients, hypertension and diabetes mellitus were the most common comorbid conditions. A large percentage of our patients had a history of smoking. Lindquist et al. also mentioned in their case series that smoking was one of the risk factors for supraglottitis [7]. Forty percent of our patients had a history of drinking cold liquids which led to symptoms of supraglottitis. The ingestion of corrosive and hot drinks are also known to be the risk factors for supraglottitis [8].

The majority of our patients presented with fever, sore throat, dysphagia, dysphonia, and leucocytosis. Most of the literature also describes these symptoms and signs to be frequent presentations [3-4, 9]. The percentage of patients presenting with stridor was $86 \%$, which is much higher than that described in the literature.

In our study, the thumb sign was positive in the majority of patients whenever a lateral neck soft tissue neck $\mathrm{x}$-ray was done. The thumb sign is due to inflammation and swelling of the epiglottis. Although a fiberoptic nasopharyngeal endoscopy is the most reliable and accurate diagnostic tool for supraglottitis, the thumb sign provides a non-invasive diagnosis with a sensitivity of $88 \%$ [10]. Recently, Fujiwara et al. published a 
In our study, a CT scan of the neck was done in $44 \%$ of the patients. A CT scan is not needed for the diagnosis of supraglottitis [12]. However, it helps to diagnose complications that include an extension of the infection into the neck and chest or formation of multiple abscesses in the oropharyngeal area [3].

In the past, $H$. influenzae was a common microorganism causing supraglottitis. As the immunization against $H$. influenzae was widespread and effective, the frequency of supraglottitis caused by other organisms is increasing. Nowadays, it is caused commonly by Streptococcus, Staphylococcus, and less frequently in immunosuppressed patients, by K. pneumoniae, E. coli, Bacteroides, anaerobes, Neisseria, MRSA, and fungi [3$4,13]$. The Streptococcus species were the most common bacteria to cause supraglottitis in our study as well, and only one patient had supraglottitis due to MRSA. Young et al., however, reported that an increasing incidence of AAS is being caused by MRSA [14].

In our patients, the common antimicrobial agent used was ceftriaxone, followed by piperacillin + tazobactam and amoxicillin + clavulanic acid, empirically (as in the majority of our patients, microbiological cultures were not taken) and after microbiological culture and sensitivities were available. The literature also suggests starting empirical treatment with third-generation cephalosporins in these patients $[1,3,9]$. Wu et al. found that third-generation cephalosporins and amoxicillin + clavulanic acid were the most frequently used antimicrobial agents in upper respiratory tract infections, including supraglottitis [15]. Adrenaline nebulization was given to $66 \%$ of our patients. It is mentioned that adrenaline nebulization helps by decreasing local tissue edema $[3,16]$.

All of our patients received steroids as adjuvant therapy due to the anti-inflammatory effect coupled with the stabilization of endothelial permeability, thus, decreasing tissue edema [17]. Recent literature suggests steroids are increasingly used in the treatment of AAS $[3,9,13,16]$.

Fifty percent of our patients did not require any airway interventions, while 39\% needed intubation. All of these intubations were difficult and required fiberoptic or video laryngoscopy. Ten percent of our patients required tracheostomy, as endotracheal intubation was impossible. Frantz et al. reported an intubation rate of $9 \%$ in supraglottitis patients, as their patients were not managed in the ICU and had milder infections [18]. In a recent study, Tapiovaara et al. found 50\% of AAS patients required intubations [19].

In our patients, $12.7 \%$ required tracheostomy to secure the airway. $\mathrm{Ng}$ et al. reported that only $0.9 \%$ of their AAS patients required tracheostomy [4]. Tapiovaara et al. reported that half of their patients required tracheostomy, whereas, in a recent meta-analysis and systematic review of AAS, Sideris et al. found that $10 \%$ of patients required airway interventions [19-20].

In our study, a majority of patients recovered without any complications. The most common complication (33\%) was the extension of supraglottic infection and abscess formation in the oropharyngeal, sublingual, and submental regions, which is known as Ludwig's angina. Sixteen percent of our patients had complete respiratory obstruction resulting in apnea.

Our patients who developed Ludwig's angina presented with fever, dysphagia, or dyspnea and required significantly higher airway interventions, as well as a significantly longer duration of symptoms and a longer stay in the ICU.

The weakness of our study is that it is a single-center, retrospective study and the majority of the patients did not have microbiological investigations. A higher sample size can also increase the power of the study.

\section{Conclusions}

AAS is a potentially life-threatening respiratory infection. In our study, the patients presenting with symptoms had higher rates of stridor. Risk factors included smoking and drinking chilled liquids. The thumb sign was helpful in diagnosis and CT of the neck was required to diagnose the complications. Although in the majority of patients a microbiological workup was not done, frequent microorganisms causing AAS were the Streptococcus species. Third-generation cephalosporins, in combination with steroids and adrenaline nebulization, were the predominant medical therapy. Critical complications included apnea and Ludwig's angina. Patients with a longer duration of symptoms had a higher chance of developing Ludwig's angina.

\section{Additional Information}

\section{Disclosures}

Human subjects: Consent was obtained by all participants in this study. Institutional Medical Research Committee, Hamad Medical Corporation, Doha issued approval MRC/562/2014. Animal subjects: All authors have confirmed that this study did not involve animal subjects or tissue. Conflicts of interest: In compliance with the ICMJE uniform disclosure form, all authors declare the following: Payment/services info: All authors have declared that no financial support was received from any organization for the 
submitted work. Financial relationships: All authors have declared that they have no financial relationships at present or within the previous three years with any organizations that might have an interest in the submitted work. Other relationships: All authors have declared that there are no other relationships or activities that could appear to have influenced the submitted work.

\section{References}

1. Navaratnam AV, Smith ME, Majeed A, McFerran DJ: Supraglottitis: a potential airway emergency that can present in primary care. Br J Gen Pract. 2015, 65:99-100. 10.3399/bigp15X683653

2. Lichtor JL, Roche Rodriguez M, Aaronson NL, Spock T, Goodman TR, Baum ED: Epiglottitis: it hasn't gone away. Anesthesiology. 2016, 124:1404-1407. https://doi.org/10.1097/ALN.0000000000001125

3. Bizaki AJ, Numminen J, Vasama JP, Laranne J, Rautiainen M: Acute supraglottitis in adults in Finland: review and analysis of 308 cases. Laryngoscope. 2011, 121:2107-2113. 10.1002/lary.22147

4. Ng HL, Sin LM, Li MF, Que TL, Anandaciva S: Acute epiglottitis in adult: a retrospective review of 106 patients in Hong Kong. Emerg Med J. 2008, 25:253-255. 10.1136/emj.2007.050153

5. Suzuki S, Yasunaga H, Matsui H, Fushimi K, Yamasoba T: Factors associated with severe epiglottitis in adults: analysis of a Japanese inpatient database. Laryngoscope. 2015, 125:2072-2078. 10.1002/lary.25114

6. Abdallah C: Acute epiglottitis: trends, diagnosis and management. Saudi J Anaesth. 2012, 6:279-281. 10.4103/1658-354X.101222

7. Lindquist B, Zachariah S, Kulkarni A: Adult epiglottitis: a case series. Perm J. 2017, 21:16-089 10.7812/TPP/16-089

8. Verhees V, Ketharanathan N, Oen IMMH, Baartmans MGA, Koopman JSHA: Beware of thermal epiglottis! A case report describing 'teapot syndrome'. BMC Anesthesiol. 2018, 18:203. 10.1186/s12871-018-0665-7

9. Cruz MGY, Almazan NA: Adult acute epiglottitis: an eight-year experience in a Philippine Tertiary Government Hospital. Philipp J Otolaryngol Head Neck Surg. 2016, 31:20-23. 10.32412/pjohns.v31i2.227

10. Takata M, Fujikawa T, Goto R: Thumb sign: acute epiglottitis. BMJ Case Rep. 2016, 2016:bcr2016214742. 10.1136/bcr-2016-214742

11. Fujiwara T, Miyata T, Tokumasu H, Gemba H, Fukuoka T: Diagnostic accuracy of radiographs for detecting supraglottitis: a systematic review and meta-analysis. Acute Med Surg. 2016, 4:190-197. 10.1002/ams2.256

12. Ramalanjaona G, Shpak M: Challenges in diagnosis of adult epiglottitis: limitation of CT scan . Emerg Med Open J. 2014, 1:1-4. 10.17140/EMOJ-1-101

13. Ramlatchan SR, Kramer N, Ganti L: Back to basics: a case of adult supraglottitis . Cureus. 2018, 10:e3475. 10.7759/cureus.3475

14. Young LS, Price CS: Complicated adult epiglottitis due to methicillin resistance staphylococcus aureus . Am J Otolaryngol. 2007, 28:441-443. 10.1016/j.amjoto.2006.11.010

15. Wu Y, Yang C, Xi H, Zhang Y, Zhou Z, Hu Y: Prescription of antibacterial agents for acute upper respiratory tract infections in Beijing, 2010-2012. Eur J Clin Pharmacol. 2016, 72:359-364. 10.1007/s00228-015-1997-6

16. Rabeea M, Al Ansari H, Al Abdulla A: An atypical cause of an epiglottis abscess. Case Rep Infect Dis. 2019, 2019:9674852. 10.1155/2019/9674852

17. Phillips JS, Innes AJ, Naik MS: Corticosteroids for supraglottitis. Br J Anaesth. 2004, 92:454-455. 10.1093/bja/aeh527

18. Frantz TD, Rasgon BM, Quesenberry CP Jr: Acute epiglottitis in adults. Analysis of 129 cases . JAMA. 1994, 272:1358-1360. 10.1001/jama.1994.03520170068038

19. Tapiovaara LK, Aro KLS, Bäck LJJ, Koskinen AIM: Comparison of intubation and tracheotomy in adult patients with acute epiglottitis or supraglottitis. Eur Arch Otorhinolaryngol. 2019, 276:3173-3177. 10.1007/s00405-019-05624-0

20. Sideris A, Holmes TR, Cumming B, Havas T: A systematic review and meta-analysis of predictors of airway intervention in adult epiglottitis. Laryngoscope. 2020, 130:465-473. 10.1002/lary.28076 